Andrea L.C. Schneider, M.D., Ph.D.

Johns Hopkins University School of Medicine

Baltimore, MD

achris13@jhmi.edu

Dan Wang, M.S.

Elizabeth Selvin, Ph.D., M.P.H.

Johns Hopkins University Bloomberg School of Public Health Baltimore, MD

\section{and Others}

A complete list of authors is available with the full text of this letter at NEJM.org.

Supported by grants from the National Institutes of Health (NIH) National Institute of Neurological Disorders and Stroke (administrative supplement to R25NS065729 to Dr. Schneider), the NIH National Institute on Aging (K24AG052573, to Dr. Gottesman), and the NIH National Institute of Diabetes and Digestive and Kidney Diseases (K24DK106414, to Dr. Selvin).
Disclosure forms provided by the authors are available with the full text of this letter at NEJM.org.

1. McMillan TM, Weir CJ, Wainman-Lefley J. Mortality and morbidity 15 years after hospital admission with mild head injury: a prospective case-controlled population study. J Neurol Neurosurg Psychiatry 2014;85:1214-20.

2. Frost RB, Farrer TJ, Primosch M, Hedges DW. Prevalence of traumatic brain injury in the general adult population: a metaanalysis. Neuroepidemiology 2013;40:154-9.

3. Corrigan JD, Selassie AW, Orman JA. The epidemiology of traumatic brain injury. J Head Trauma Rehabil 2010;25:72-80.

4. Wilmoth $\mathrm{K}$, LoBue C, Clem MA, et al. Consistency of traumatic brain injury reporting in older adults with and without cognitive impairment. Clin Neuropsychol 2018;32:524-9.

5. Corrigan JD, Bogner J. Initial reliability and validity of the Ohio State University TBI Identification Method. J Head Trauma Rehabil 2007;22:318-29.

DOI: 10.1056/NEJMc1808550

\title{
Systemic AA Amyloidosis Caused by Inflammatory Hepatocellular Adenoma
}

TO THE EDITOR: Amyloid A (AA) systemic amyloidosis is a complication of chronic inflammatory diseases that is caused by the deposition of insoluble aggregates of cleaved $\mathrm{N}$-terminal fragments of serum amyloid A (SAA) protein in tissues and organs throughout the body. ${ }^{1}$ Under physiologic conditions, SAA protein is produced by hepatocytes during the acute inflammatory phase in response to various cytokines such as interleukin-6. SAA is also overexpressed by neoplastic hepatocytes in inflammatory hepatocellular adenomas, a specific molecular subtype of benign liver tumors. ${ }^{2,3}$

Here, we describe a 49-year-old female patient who presented with diarrhea, rectal bleeding, and leg edema and received a diagnosis of systemic AA amyloidosis. Clinical characteristics of the patient are provided in Table S1 in the Supplementary Appendix, available with the full text of this letter at NEJM.org. An inflammatory hepatocellular adenoma was identified and resected, resulting in the improvement of amyloidosisrelated symptoms, with progressive normalization of the serum C-reactive protein (Fig. 1A) and SAA levels. Unfortunately, the patient's kidney function did not improve, and she died 18 months after surgery from fulminant septic shock.

Whole-genome sequencing of the inflammatory hepatocellular adenoma revealed a complex structural rearrangement on chromosome 7 with an inversion leading to the truncation of the IL6 $3^{\prime}$ untranslated region (3'UTR), which comprises key sequences involved in the degradation of this unstable transcript (Fig. 1, and Tables S2 and S3
Figure 1 (facing page). Biologic, Imaging, Pathological, and Molecular Findings in Our Patient.

As shown in Panel A, liver resection was followed by a clinically significant decrease in serum levels of C-reactive protein (CRP) (top), and magnetic resonance imaging showed a heterogeneous, $5-\mathrm{cm}$ liver mass at the tip of segment $\mathrm{VI}$ on $\mathrm{T}_{2}$-weighted sequences (bottom, arrow). As shown in Panel B, microscopic examination of the resected specimen revealed a well-differentiated tumor with massive amyloid deposits (arrows) (subpanel a, Congo red staining, low magnification) and typical yellow-green birefringence under polarized light (subpanel b, low magnification). Immunohistochemical tests showed positive staining for serum amyloid A (SAA) protein in neoplastic hepatocytes (black arrows) and amyloid deposits (red arrows) in the tumor ( $T$ ) sample (subpanel c, high magnification); SAA expression was also observed in the adjacent nontumorous (NT) parenchyma (subpanel d, high magnification). CRP was expressed in the tumor (T) (subpanel e, low magnification) (neoplastic hepatocytes, black arrows) and the adjacent NT liver tissue (subpanel $f$, high magnification) (non-neoplastic hepatocytes, black arrows). As shown in Panel C, whole-genome sequencing of the tumor sample obtained from the patient revealed a cluster of somatic structural rearrangements at the ILG locus on chromosome 7. The blue line in the outer circle indicates copy number, and structural rearrangements are indicated in the inner circle. Red indicates deletion, blue inversion, gray interchromosomal translocation, and black classic chromatin staining of the cytobands. As shown in Panel D, although overexpression of ILG was specific to the tumor sample, the genes $S A A 1$ and $C R P$ were also massively overexpressed in the adjacent liver tissue, as compared with normal liver tissue. FPKM denotes fragments per kilobase of exon per 1 million reads in the RNA sequencing experiment. As shown in Panel $\mathrm{E}$, chromosomal inversion with one breakpoint located in the $3^{\prime}$ untranslated region (UTR) of the gene I $L G$ and the other breakpoint in an intergenic region at $7 p 14.1$ led to the massive overexpression of an interleukin- 6 transcript lacking regulatory $3^{\prime} U T R$ elements in the patient's tumor (top, structural rearrangement identified by means of whole-genome sequencing; bottom, gene expression and transcript structure identified by means of RNA sequencing). AU denotes adenylate-uridylate. 
in the Supplementary Appendix). RNA sequencing consistently showed a massive overexpression of an interleukin- 6 transcript lacking all 3' regulatory elements; this overexpression was limited to the tumor area. However, in contrast to classic inflammatory hepatocellular adenoma, we observed overexpression of SAA in both the tumor and nontumorous liver. Amyloid deposits were also identified in the adenoma and the adjacent parenchyma (Fig. 1). These results support an autonomous production of interleukin-6 by the inflammatory hepatocellular adenoma with activation of a paracrine Janus kinase-signal transducers and activators of transcription 3

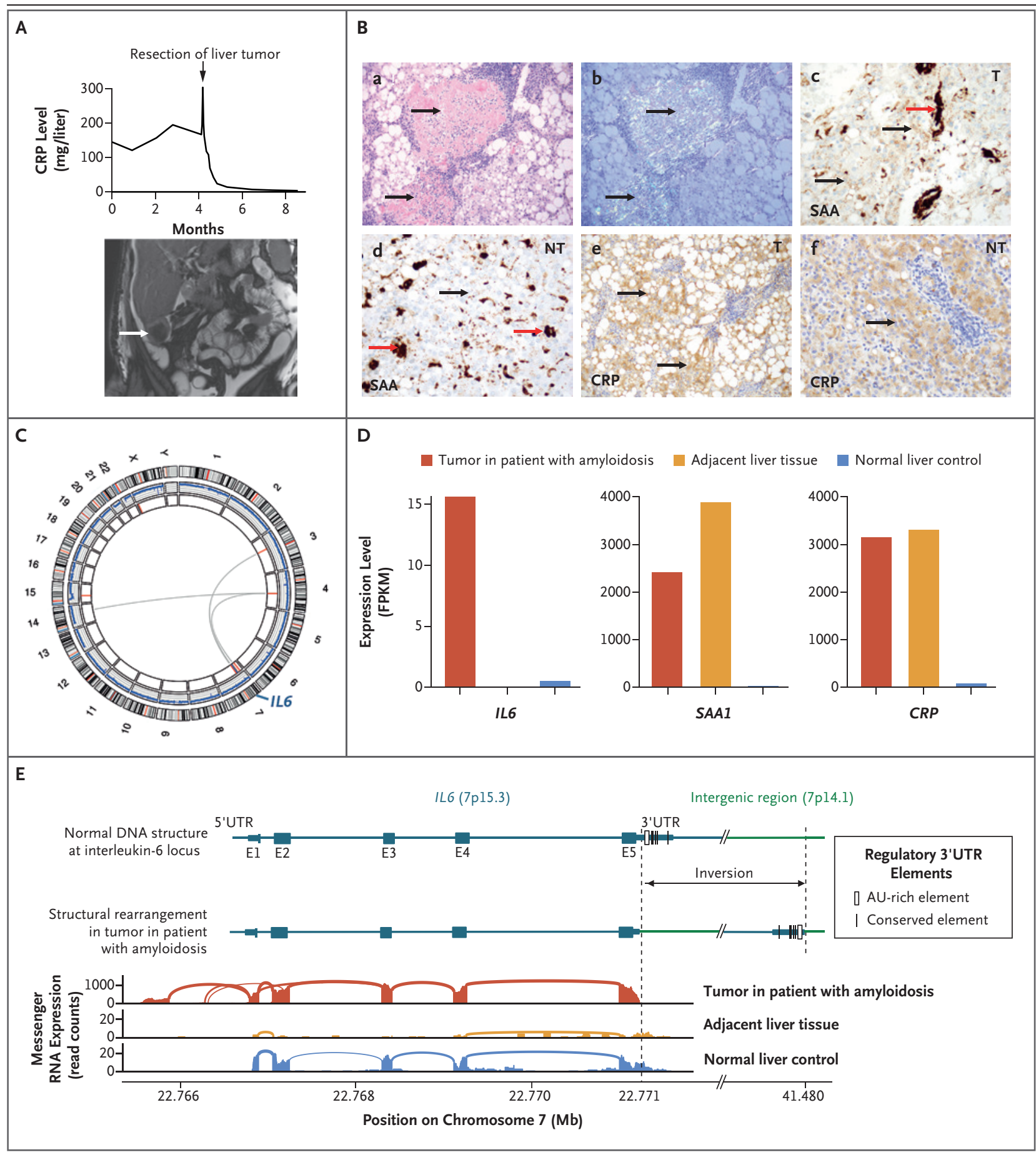

N ENGLJ MED 379;12 NEJM.ORG SEPTEMBER 20, 2018

The New England Journal of Medicine 
(JAK-STAT3) pathway in the liver (Fig. S1 in the Supplementary Appendix).

In the literature, we identified eight additional patients with both AA amyloidosis and hepatocellular adenomas. In at least six of these patients, amyloid-related symptoms also improved after surgical resection of the liver tumor (Table S4 in the Supplementary Appendix). We reviewed the liver histologic findings of two patients from the literature and identified features that were similar to those of our patient; inflammatory hepatocellular adenoma with SAA expression by the tumor and the adjacent parenchyma was indeed identified in both cases from the literature (Fig. S2 in the Supplementary Appendix)., ${ }^{4,5} \mathrm{Bi}-$ refringent, SAA-positive amyloid deposits were also identified in the adenomas and adjacent liver tissue.

In conclusion, rare cases of inflammatory hepatocellular adenoma can induce massive SAA production by the tumor and nontumorous liver, leading to systemic AA amyloidosis. Molecular analyses identified a somatic rearrangement of the IL6 3'UTR leading to an autocrine interleukin-6 secretion by the tumor. We speculate that this subset of AA amyloidosis may be treatable by surgical resection of the liver tumor.

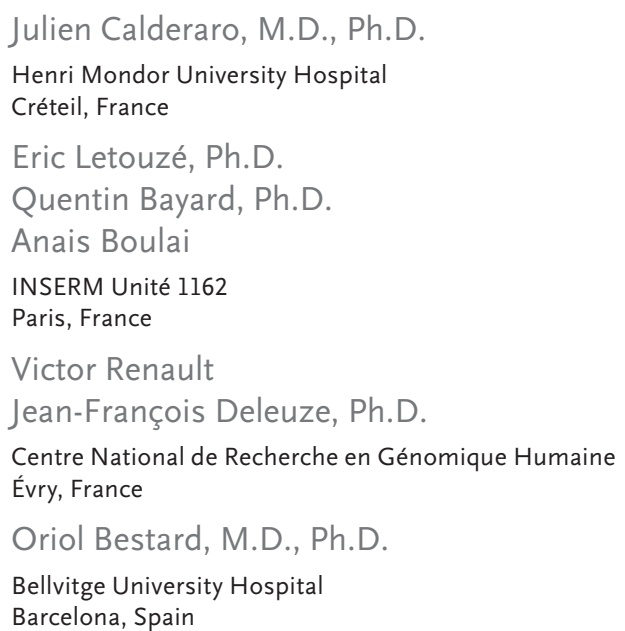

Dominique Franco, M.D., Ph.D.

Antoine Béclère University Hospital

Clamart, France

Elie-Serge Zafrani, M.D.

Henri Mondor University Hospital

Créteil, France

Jean-Charles Nault, M.D., Ph.D.

INSERM Unité 1162

Paris, France

Michel Moutschen, M.D., Ph.D.

Liege University Hospital

Liege, Belgium

Jessica Zucman-Rossi, M.D., Ph.D.

INSERM Unité 1162

Paris, France

jessica.zucman-rossi@inserm.fr

Drs. Calderaro, Letouzé, and Bayard contributed equally to this letter.

Supported by the Institut National du Cancer with the International Cancer Genome Consortium (ICGC Liver CancerFrance project), INSERM with the "Cancer et Environnement" (plan Cancer) and Heterogeneity of Colorectal and Liver Cancer (HETCOLI) projects (Tumor Heterogeneity and Ecosystem program), the Ligue Nationale contre le Cancer (Equipe Labellisée), Labex OncoImmunology Investissement d'Avenir, Coup d'Elan de la Fondation Bettencourt-Schueller, the Site de Recherche Intégrée sur le Cancer-Cancer Research and Personalized Medicine (SIRIC CARPEM), Fondation Mérieux, Cancéropôle Ile de France (exhauTrans project), and Instituto de Salud Carlos III (Spanish competitive grants FIS PI16/01321 and INT15/00112, to Dr. Bestard) through the European Regional Development Fund for research.

Disclosure forms provided by the authors are available with the full text of this letter at NEJM.org.

1. Wechalekar AD, Gillmore JD, Hawkins PN. Systemic amyloidosis. Lancet 2016;387:2641-54.

2. Nault JC, Couchy G, Balabaud C, et al. Molecular classification of hepatocellular adenoma associates with risk factors, bleeding, and malignant transformation. Gastroenterology 2017; 152(4):880-894.e6.

3. Rebouissou S, Amessou M, Couchy G, et al. Frequent inframe somatic deletions activate gp130 in inflammatory hepatocellular tumours. Nature 2009;457:200-4.

4. Bestard Matamoros O, Poveda Monje R, Ibernon Vilaró M, Carrera Plans M, Grinyó Boira JM. Systemic AA amyloidosis induced by benign neoplasms. Nefrologia 2008;28:93-8. (In Spanish.)

5. Fievet P, Sevestre H, Boudjelal M, et al. Systemic AA amyloidosis induced by liver cell adenoma. Gut 1990;31:361-3.

DOI: 10.1056/NEJMc1805673

\section{Sodium Thiosulfate and Cisplatin-Induced Hearing Loss}

TO THE EDITOR: Brock and colleagues (June 21 issue) ${ }^{1}$ report the results of International Liver Tumor Strategy Group (SIOPEL) 6, a randomized, controlled trial of sodium thiosulfate for protec- tion from cisplatin-induced hearing loss. In addition to confirming the efficacy of sodium thiosulfate, as previously reported in the Children's Oncology Group ACCL0431 trial, ${ }^{2}$ SIOPEL 6 pro- 